\title{
Serum levels of PSA, ALP, ICTP, and BSP in prostate cancer patients and the significance of ROC curve in the diagnosis of prostate cancer bone metastases
}

\author{
R.J. Wei ${ }^{1}$, T.Y. Li ${ }^{2}$, X.C. Yang ${ }^{1}$, N. Jia ${ }^{1}$, X.L. Yang ${ }^{3}$ and H.B. Song ${ }^{4}$ \\ ${ }^{1}$ Department of Urology Surgery, The Affiliated Hospital of Hebei University, \\ Baoding, Hebei, China \\ ${ }^{2}$ Department of Urology Surgery, Medical College of Hebei University, \\ Baoding, Hebei, China \\ ${ }^{3}$ Department of Urology Surgery, Hebei University, Baoding, Hebei, China \\ ${ }^{4}$ Department of Urology Surgery, Quyang County Hospital of Baoding, \\ Baoding, Hebei, China \\ Corresponding author: R.J. Wei \\ E-mail: weiruojin11@163.com
}

Genet. Mol. Res. 15 (2): gmr.15027707

Received September 9, 2015

Accepted December 14, 2015

Published June 3, 2016

DOI http://dx.doi.org/10.4238/gmr.15027707

\begin{abstract}
Bone metastasis is a common complication in prostate cancer patients that can cause bone pain and pathological fracture. This study tested serum levels of prostate specific antigen (PSA), alkaline phosphatase (ALP), bone sialoprotein (BSP), collagen type I pyridine crosslinking peptide (ICTP) in prostate cancer patients and the significance of the receiver operator characteristic (ROC) curve in the diagnosis of prostate cancer bone metastases. Eighty-three prostate cancer patients were enrolled including 42 in the bone metastases group and 41 in the non-bone metastases group. Serum levels of BSP, ALP, ICTP, and PSA were highest in the bone metastases group followed by the non-bone metastases group, hyperplasia group, and then the control
\end{abstract}


group $(\mathrm{P}<0.05)$. Based on Gleason score, serum levels were highest in the poorly differentiated group followed by moderately differentiated and well-differentiated groups $(\mathrm{P}<0.05)$. ROC curve analysis revealed that the diagnostic efficiency of the biomarkers in turn was BSP, PSA, ICTP, and ALP. The sensitivity of BSP, ALP, ICTP, and PSA in the diagnosis of prostate cancer bone metastases were 80.95, 57.14, 69.05, $71.43 \%$, respectively, and the specificity of the same markers were $72.80,64.80,76.80$, and $88.80 \%$, respectively. Combined detection of the four markers improved sensitivity to $97.62 \%$ and the negativepredictive value increased to $97.60 \%$. PSA + BSP showed the best efficiency when combining two markers. In conclusion, serum levels of BSP, ALP, ICTP, and PSA increased in patients with bone metastases, and combined detection of all markers could improve the positivepredictive value.

Key words: Prostate cancer; Bone metastases; ICTP; PSA; BSP; ALP

\section{INTRODUCTION}

Prostate cancer is a common malignant tumor in the urinary system with increased incidence and mortality in recent years (Chang et al., 2015; Koo et al., 2015). Bone is a common target of distant metastases in prostate cancer (Huang et al., 2015; Mai et al., 2015). The incidence of bone metastases in the process of prostate cancer development is about $65-70 \%$, while it is found in $85-100 \%$ in patients who have died from prostate cancer (Jadaan et al., 2015; Takagi et al., 2015). Due to lack of symptoms in the onset of prostate cancer or early bone metastasis, most patients see their physician only once bone metastases have formed and produce symptoms. Prostate cancer bone metastases may lead to pathological fracture and bone pain that seriously affect quality of life and prognosis. Therefore, early diagnosis and treatment of prostate cancer bone metastases is of great significance. Currently, radionuclide bone imaging is commonly used for prostate cancer bone metastasis diagnosis and monitoring with high sensitivity. However, its disadvantages include low specificity, high cost, and the radioactive harm to patients and staff (Luna et al., 2015; Nguyen-Nielsen et al., 2015).

The detection of prostate cancer-related serum markers has increased following increased research on prostate cancer bone metastasis. Compared to the bone scan, serum markers showed advantages in their reproducibility, non-invasiveness, and relatively low cost. Serum prostate specific antigen (PSA) level can predict the lesion range of prostate cancer; PSA and alkaline phosphatase (ALP) are important predictors for bone metastasis (Kitajima et al., 2014; Pasoglou et al., 2015); serum bone sialoprotein (BSP) levels reflect the process of bone resorption and bone cell activity; and bone resorption marker collagen type I pyridine crosslinking peptide (ICTP) indicates bone cell function and bone absorption rate that has certain diagnostic significance in bone metastases (Luna et al., 2015; Pasoglou et al., 2015). This study investigated serum PSA, ALP, BSP, and ICTP levels in prostate cancer patients and the significance of receiver operating characteristic (ROC) curve in the diagnosis of prostate cancer bone metastasis to provide the basis for early diagnosis and treatment of bone metastases. 


\section{MATERIAL AND METHODS}

\section{Clinical information}

Eighty-three patients with prostate cancer were enrolled between February 2011 and March 2014 in the Affiliated Hospital of Hebei University. Another 42 patients with benign prostate hyperplasia were selected as the hyperplasia group and 42 subjects who were deemed healthy at a routine physical examination were treated as the control group. All patients were diagnosed by pathology or cytology examination. Gleason score was applied for evaluation including 26 well-differentiated cases (score 2-4), 36 moderately differentiated cases (score $5-6$ ), and 21 poorly differentiated cases (score 7-10). The patients were further divided into bone metastases group (42 cases) and non-bone metastases group (41 cases). This study was approved by the hospital Ethics Committee and all participants provided written informed consent. The average age of patients with or without bone metastases was $63.6 \pm 4.7$ or $65.2 \pm$ 5.3 years old, respectively. The mean age in the hyperplasia and normal control was $65.4 \pm 5.2$ and $64.7 \pm 4.4$ years old, respectively. The general clinical characteristics of the four groups showed no statistical difference $(\mathrm{P}>0.05)$.

\section{Detection method}

No subjects received puncture or digital rectal examination at least 3 days before blood collection. Peripheral venous blood was extracted and centrifuged at 14,000 $\mathrm{g}(\mathrm{r}=10$ $\mathrm{cm}, 3500 \mathrm{rpm}$ ) for $15 \mathrm{~min}$. The serum was analyzed within $2 \mathrm{~h}$. The interval between bone imaging examination and blood collection was less than 3 days. Serum BSP and ICTP levels were detected by enzyme-linked immunosorbent assay (ELISA) (ADL Company, USA). ALP was tested by the velocity method (Nanjing Jiancheng Biological Technology Co., Ltd., Nanjing China) and PSA level was determined by electrochemical immunoluminescence (Nanjing Jiancheng Biological Technology Co., Ltd.).

\section{Evaluation of factors}

The ROC curve was used to evaluate the significance of BSP, ALP, ICTP, and PSA levels in the diagnosis of prostate cancer bone metastases. Yoden index $\mathrm{J}$ was calculated as $\mathrm{J}=$ sensitivity + specificity -1 . The best critical point was selected as the largest point of tangency of the Yonden index. ALP > $115 \mathrm{U} / \mathrm{L}, \mathrm{ICTP}>4.3 \mathrm{U} / \mathrm{L}, \mathrm{PSA}>20 \mu \mathrm{g} / \mathrm{L}$, and BSP $>30.15 \mu \mathrm{g} / \mathrm{L}$ were treated as positive. The judgment method used for multiple detection was if any one of the markers appeared positive was deemed positive, while all of the markers deemed negative were considered negative (Moslehi et al., 2013).

\section{Statistical analysis}

All the statistical analysis was performed on the SPSS19.0 software (Chicago, USA). The chi-square test and the chi-square correction inspection were used for rate comparison. The Kolmogorov-Smirnov test was applied for normality test. The measured data are reported as means \pm standard deviation. Analysis of variance (ANOVA) and least significant difference (LSD) tests were used for mean comparison. ROC curve was applied to evaluate 
the significance of BSP, ALP, ICTP, and PSA on prostate cancer bone metastasis. Logistic regression model was established for the $\mathrm{z}$-test in combined detection comparison. $\mathrm{P}<0.05$ was considered as statistically significant.

\section{RESULTS}

\section{Comparison of serum BSP, ALP, ICTP, and PSA levels in each group}

As shown in Table 1, serum BSP, ALP, ICTP, and PSA levels were highest in the bone metastases group followed by non-bone metastases group, hyperplasia group, and control group $(\mathrm{P}<0.05)$.

Table 1. Serum BSP, ALP, ICTP, and PSA expression levels (means \pm SD).

\begin{tabular}{l|c|c|c|c|c}
\hline Group & Cases & BSP $(\mu \mathrm{g} / \mathrm{L})$ & ALP $(\mathrm{U} / \mathrm{L})$ & ICTP $(\mu \mathrm{g} / \mathrm{L})$ & PSA $(\mu \mathrm{g} / \mathrm{L})$ \\
\hline Control & 42 & $7.51 \pm 1.31$ & $57.96 \pm 4.79$ & $1.74 \pm 0.36$ & $6.44 \pm 2.91$ \\
\hline Hyperplasia & 42 & $8.11 \pm 1.47^{*}$ & $64.86 \pm 9.21^{*}$ & $2.33 \pm 0.45^{*}$ & $7.78 \pm 3.19^{*}$ \\
\hline Non-bone metastases & 41 & $9.23 \pm 3.12^{* \Delta}$ & $70.34 \pm 11.23^{*} \&$ & $3.93 \pm 1.95^{*} \Delta$ & $19.47 \pm 7.64^{*}$ \\
\hline Bone metastases & 42 & $38.93 \pm 9.12^{* \triangle \&}$ & $534.61 \pm 72.38^{*} \&$ & $14.87 \pm 2.31^{*} \&$ & $162.21 \pm 26.17^{* \Delta \&}$ \\
\hline F value & - & 32.323 & 21.336 & 36.991 & 13.392 \\
\hline P value & - & 0.000 & 0.000 & 0.000 & 0.000 \\
\hline
\end{tabular}

$* \mathrm{P}<0.05$, compared with control; $\triangle \mathrm{P}<0.05$, compared with hyperplasia group; ${ }^{\&} \mathrm{P}<0.05$, compared with nonbone metastases group.

\section{Comparison of serum BSP, ALP, ICTP, and PSA levels using Gleason score}

Based on the Gleason score, serum BSP, ALP, ICTP, and PSA levels were highest in poorly differentiated group, followed by moderately differentiated group then welldifferentiated group $(\mathrm{P}<0.05$; Table 2).

Table 2. Comparison of serum BSP, ALP, ICTP, and PSA expression levels using the Gleason score (means \pm SD).

\begin{tabular}{l|c|c|c|c|c}
\hline Group & Cases & BSP $(\mu \mathrm{g} / \mathrm{L})$ & ALP $(\mathrm{U} / \mathrm{L})$ & ICTP $(\mu \mathrm{g} / \mathrm{L})$ & PSA $(\mu \mathrm{g} / \mathrm{L})$ \\
\hline Well-differentiated & 26 & $27.65 \pm 4.37$ & $231.46 \pm 14.52$ & $9.32 \pm 2.11$ & $56.78 \pm 11.21$ \\
\hline Moderately differentiated & 36 & $36.61 \pm 4.28^{*}$ & $407.25 \pm 15.27^{*}$ & $12.67 \pm 3.14^{*}$ & $123.64 \pm 22.36^{*}$ \\
\hline Poorly differentiated & 21 & $48.93 \pm 5.12^{* \Delta}$ & $565.34 \pm 21.23^{* \Delta}$ & $18.13 \pm 4.95^{*} \Delta$ & $189.23 \pm 42.34^{* \Delta}$ \\
\hline F value & - & 3.570 & 7.708 & 4.143 & 4.717 \\
\hline P value & - & 0.038 & 0.002 & 0.024 & 0.015 \\
\hline
\end{tabular}

$* \mathrm{P}<0.05$, compared with well-differentiated group; ${ }^{\triangle} \mathrm{P}<0.05$, compared with moderate differentiation group. High differentiated group $v s$ low differentiated group, $* \mathrm{P}<0.05$, moderate differentiated group $v s$ low differentiated group, $\triangle \mathrm{P}<0.05$.

\section{ROC curve analysis of serum levels of BSP, ALP, ICTP, and PSA}

The ROC curve was established based on serum levels of factors in control and bone metastase groups. All factors were useful in the diagnosis of bone metastasis but BSP was most useful in terms of diagnostic value, followed by PSA, ICTP, and ALP (Figure 1 and Table 3). 


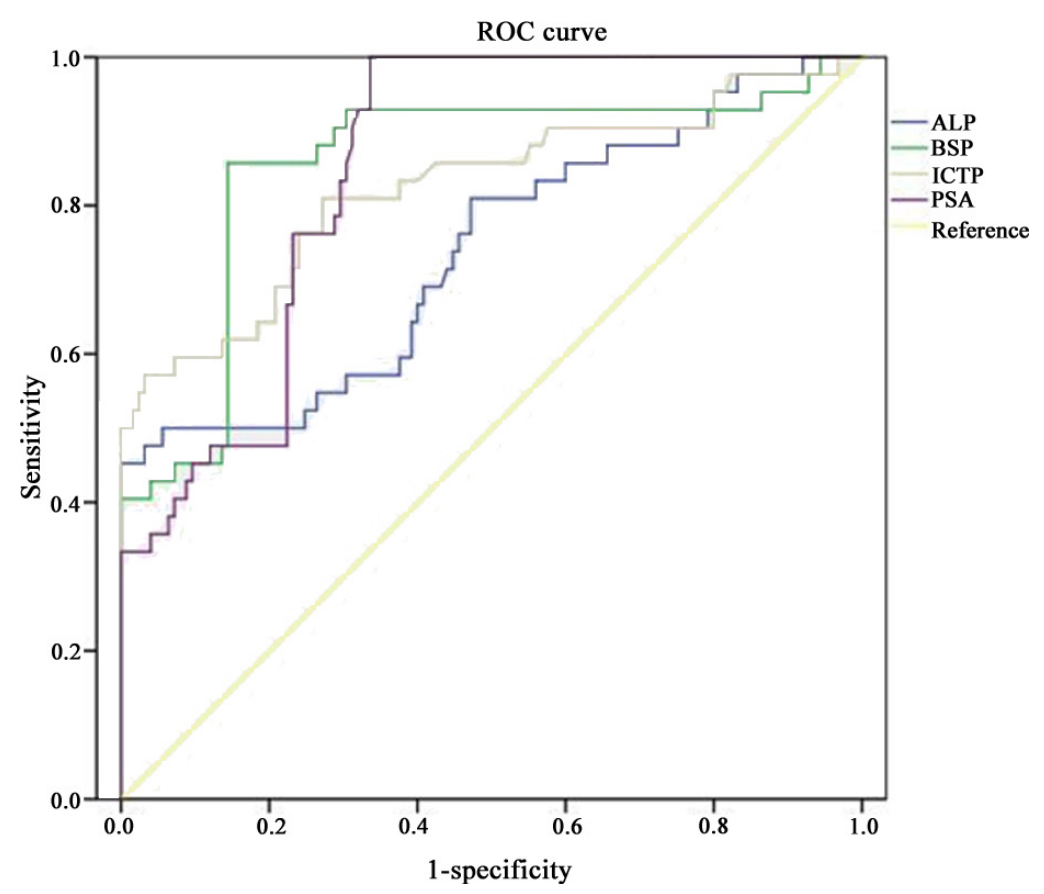

Figure 1. ROC curve of sensitivity versus specificity of BSP, ALP, ICTP, and PSA in prostate cancer bone metastases. The reference curve is also shown.

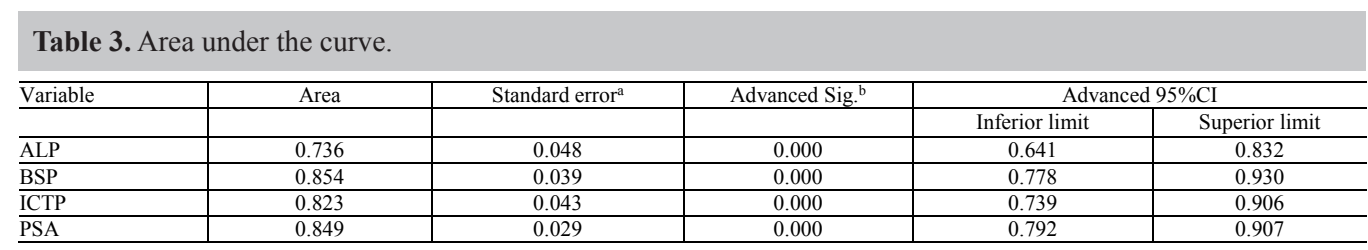

${ }^{\mathrm{a}}$ Under nonparametric hypothesis; ${ }^{b}$ null hypothesis: solid area $=0.5$.

\section{Comparison of detection of BSP, ALP, ICTP, and PSA}

The BSP, ALP, ICTP, and PSA levels in the control and bone metastase groups together with ROC curve analysis were used to calculate the Yoden index $\mathrm{J}(\mathrm{J}=$ sensitivity + specificity -1). The best critical point was selected as the largest point of tangency of the Yonden index. ALP $>115 \mathrm{U} / \mathrm{L}, \mathrm{ICTP}>4.3 \mathrm{U} / \mathrm{L}, \mathrm{PSA}>20 \mu \mathrm{g} / \mathrm{L}$, and BSP $>30.15 \mu \mathrm{g} / \mathrm{L}$ were treated as positive. The sensitivity of BSP, ALP, ICTP, and PSA in the diagnosis of prostate cancer bone metastases were $80.95,57.14,69.05$, and $71.43 \%$, respectively. The specificity of BSP, ALP, ICTP, and PSA in the diagnosis of prostate cancer bone metastases were 72.80, $64.80,76.80$, and $88.80 \%$, respectively. BSP showed higher sensitivity than PSA, ICTP, and ALP. While BSP showed higher sensitivity, PSA presented higher specificity $(\mathrm{P}<0.05$; Table 4). 
Table 4. Comparison of detection of BSP, ALP, ICTP, and PSA.

\begin{tabular}{l|c|c|c}
\hline Index & Positive critical value & Sensitivity (\%) & Specificity (\%) \\
\hline BSP & $>30.15 \mu \mathrm{g} / \mathrm{L}$ & $80.95(34 / 42)$ & $72.80(91 / 125)$ \\
\hline ALP & $>115 \mathrm{U} / \mathrm{L}$ & $57.14(24 / 42)$ & $64.80(81 / 125)$ \\
\hline ICTP & $4.3 \mathrm{U} / \mathrm{L}$ & $69.05(29 / 42)$ & $76.80(96 / 125)$ \\
\hline PSA & $20 \mu \mathrm{g} / \mathrm{L}$ & $71.43(30 / 42)$ & $88.80(111 / 125)$ \\
\hline$\chi^{2}$ & - & 25.929 & 133.128 \\
\hline P value & - & $<0.05$ & $<0.05$ \\
\hline
\end{tabular}

\section{Combined detection of BSP, ALP, ICTP, and PSA}

The combined detection of all four markers improved sensitivity to $95.24 \%$ and negative-predictive value to $98.06 \%$. The area under the curve for the combined detection of all four markers was $0.966(95 \%$ confidence interval $(\mathrm{CI})=0.919-0.999)$. PSA + BSP and ICTP + BSP showed better efficiency than the combined detection of all four markers (Figure 2, Tables 5 and 6 ).

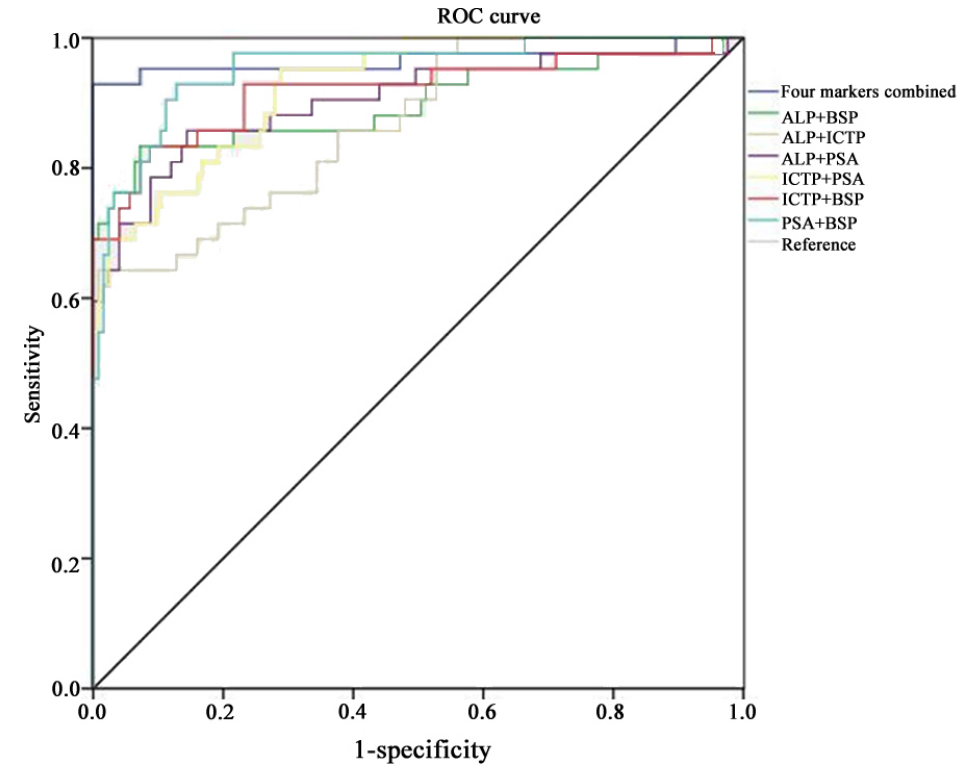

Figure 2. ROC curve of sensitivity versus specificity of combinations of BSP, ALP, ICTP, and PSA. The reference curve and all four markers combined are also shown.

\section{Table 5. Area under the curve of combined detection.}

\begin{tabular}{|c|c|c|c|c|c|}
\hline$\overline{\text { Variable }}$ & Area & Standard error ${ }^{\mathrm{a}}$ & Advanced Sig. & \multicolumn{2}{|c|}{ Advanced $95 \% \mathrm{CI}$} \\
\hline & & & & Inferior limit & Superior limit \\
\hline Four markers combined & 0.966 & 0.024 & 0.000 & 0.919 & 0.999 \\
\hline $\mathrm{ALP}+\mathrm{BSP}$ & 0.899 & 0.036 & 0.000 & 0.828 & 0.969 \\
\hline$\overline{\mathrm{ALP}}+\mathrm{ICTP}$ & 0.868 & 0.033 & 0.000 & 0.804 & 0.932 \\
\hline ALP + PSA & 0.904 & 0.033 & 0.000 & 0.840 & 0.968 \\
\hline ICTP + PSA & 0.925 & 0.022 & 0.000 & 0.882 & 0.968 \\
\hline$\overline{\mathrm{ICTP}}+\mathrm{BSP}$ & 0.919 & 0.031 & 0.000 & 0.858 & 0.980 \\
\hline PSA + BSP & 0.953 & 0.019 & 0.000 & 0.916 & 0.990 \\
\hline
\end{tabular}

${ }^{\mathrm{a} U n d e r}$ nonparametric hypothesis; ${ }^{\mathrm{b}}$ null hypothesis: solid area $=0.5$.

Genetics and Molecular Research 15 (2): gmr.15027707 
Table 6. Comparison of the results of combined detection of BSP, ALP, ICTP, and PSA.

\begin{tabular}{l|c|c}
\hline Index & Sensitivity (\%) & Specificity (\%) \\
\hline Four markers combined & $95.24(40 / 42)$ & $79.20(101 / 125)$ \\
\hline ALP + ICTP & $73.81(31 / 42)$ & $84.80(106 / 125)$ \\
\hline ALP + PSA & $80.95(34 / 42)$ & $87.20(109 / 125)$ \\
\hline ALP + BSP & $78.57(33 / 42)$ & $83.20(104 / 125)$ \\
\hline ICTP + PSA & $88.10(37 / 42)$ & $92.00(115 / 125)$ \\
\hline ICTP + BSP & $83.33(35 / 42)$ & $85.60(107 / 125)$ \\
\hline PSA + BSP & $90.48(38 / 42)$ & $90.40(113 / 125)$ \\
\hline
\end{tabular}

\section{DISCUSSION}

The early stages of bone metastasis in prostate cancer have no significant clinical features. Bone X-rays show no sign of bone destruction as it is a low-sensitivity technique. Magnetic resonance imaging and radionuclide bone imaging have higher sensitivity, but come with the dangers of radioactive exposure and high cost. Due to the hidden onset of prostate cancer, bone metastasis shows no obvious symptoms in early stages. Bone metastases may lead to pathological fracture and bone pain that can seriously affect prognosis and quality of life. Therefore, early diagnosis and treatment of prostate cancer bone metastases is of great significance. Serum bone markers have the advantages of being repeatable, non-invasive, and low in cost. Also, they can reflect the bone metabolism of the entire body. Searching for effective and sensitive serum indicators is important, as prostate cancer patients may present bone pain or pathological fracture. Gleason score $\geq 8$, PSA $\geq 20 \mu \mathrm{g} / \mathrm{L}$, and ALP elevation are risk factors for prostate cancer bone metastases (Kamiya et al., 2010; Moslehi et al., 2013). Bone metastases destroy the balance between osteoblast-mediated bone formation and osteoclast-mediated bone resorption (Lein et al., 2007). BSP is a non-collagenous protein that can promote bone metastases in breast cancer. Serum BSP has certain significance for lung cancer bone metastases as well (Ramankulov et al., 2007; Wang et al., 2013). This study investigated the role of BSP in evaluating prostate cancer bone metastasis. The result showed that BSP level in patients with bone metastasis was significantly higher than the patients with no bone metastasis. ROC curve analysis revealed that the sensitivity and specificity for BSP in diagnosing prostate cancer bone metastasis were 80.95 and $72.80 \%$, respectively. This indicated that BSP values are a sensitive and valuable option for diagnosis of prostate cancer bone metastases.

Bone metabolic markers are mainly produced by bone matrix metabolism and bone cell secretion. Cytokines may act on osteoclasts in malignant tumor bone metastasis, leading to normal bone matrix damage and increases in abnormal bone metabolic marker levels. The vertebral venous plexus is an important channel for the superior and inferior vena cava that mainly gathers bone marrow vein and vertebral bone vein. Extensive traffic between prostate cancer venous return and vertebral vein is the physiological basis of bone metastases. PSA and ALP are important predictors for bone metastases (Kitajima et al., 2014; Pasoglou et al., 2015). As a specific biomarker of prostate cancer that is widely used in the clinic, serum PSA can predict the lesion range of prostate cancer and is associated with prostate cancer metastasis and prognosis. The bone metabolic state in bone metastases has no direct correlation with PSA and needs combined detection for diagnosis (Jung et al., 2004; Kataoka et al., 2006). There have been numerous studies focused on bone markers in prostate cancer bone metastasis, but the accuracy in early diagnosis was not consistent (Kataoka et al., 2006; Szot et al., 2014). 
Osteolysis and osteogenesis lesions exist in prostate cancer bone metastases. Imaging data showed that osteogenesis lesions were prominent in prostate cancer bone metastases, indicating the significance of bone resorption and bone formation serum markers in the diagnosis of prostate cancer bone metastases. Bone resorption marker ICTP is a specific product in the process of type I collagen degradation that can reflect bone cell function and bone resorption rate. Studies have shown that ICTP can reflect the degree of bone metastases in malignancy. It showed a positive correlation with the number of bone metastatic lesions and increased following bone metastasis upstaging. It is also important for malignant tumor curative effect evaluation, clinical stage, and prognosis (Lojanapiwat et al., 2014; Fizazi et al., 2015).

This study result showed that serum BSP, ALP, ICTP, and PSA levels were elevated in prostate cancer patients and were higher in the bone metastasis group compared with nonbone metastasis group. Upon examination of the Gleason score, their levels in the poorly differentiated group were higher than in the well-differentiated group. Gleason scores are important to evaluate postoperative staging, and serum BSP, ALP, ICTP, and PSA levels correlate with tumor differentiation degree. Prostate cancer epithelial cell hyperplasia, blood barrier lesions, and abnormal enhancement of local blood transportation result in abnormal bone marker elevation. ROC curve analysis showed that the diagnostic efficiency of each marker in turn was BSP, PSA, ICTP, and ALP. Based on single indicator evaluation, BSP presented high sensitivity while PSA and ICTP presented high specificity. ALP only increased significantly after widespread bone metastasis and its sensitivity is low. The combined detection of all four markers improved sensitivity to $97.62 \%$ and the negative predictive value increased to $97.60 \%$. Dual combination of PSA + BSP and ICTP + BSP showed better efficiency, suggesting that combined detection can reflect the bone metabolic state in early stages and enhance the sensitivity and specificity. Serum biomarkers reflect early bone metabolic changes and combined detection of bone markers can contribute to the early diagnosis of prostate cancer bone metastasis. In conclusion, serum BSP, ALP, ICTP, and PSA levels are increased in prostate cancer bone metastasis, and combined detection of more than one marker could improve the positive predictive value.

\section{Conflicts of interest}

The authors declare no conflict of interest.

\section{ACKNOWLEDGMENTS}

We thank the anonymous reviewers for reviewing this manuscript.

\section{REFERENCES}

Chang CC, Lee YC, Tsai HW, Yii SC, et al. (2015). Diagnostic role of serum free-to-total prostate specific antigen (PSA) ratio in prostate cancer with serum total concentration of PSA below $4 \mathrm{ng} / \mathrm{mL}$. Asian Pac. J. Cancer Prev. 16: 52615264. http://dx.doi.org/10.7314/APJCP.2015.16.13.5261

Fizazi K, Massard C, Smith M, Rader M, et al. (2015). Bone-related parameters are the main prognostic factors for overall survival in men with bone metastases from castration-resistant prostate cancer. Eur. Urol. 68: 42-50. http://dx.doi. org/10.1016/j.eururo.2014.10.001

Huang Y, Jin C, Hamana T, Liu J, et al. (2015). Overexpression of FGF9 in prostate epithelial cells augments reactive stroma formation and promotes prostate cancer progression. Int. J. Biol. Sci. 11: 948-960. http://dx.doi.org/10.7150/ijbs.12468 
Jadaan DY, Jadaan MM and McCabe JP (2015). Cellular plasticity in prostate cancer bone metastasis. Prostate Cancer 2015: 651580. http://dx.doi.org/10.1155/2015/651580

Jung K, Lein M, Stephan C, Von Hösslin K, et al. (2004). Comparison of 10 serum bone turnover markers in prostate carcinoma patients with bone metastatic spread: diagnostic and prognostic implications. Int. J. Cancer 111: 783-791. http://dx.doi.org/10.1002/ijc.20314

Kamiya N, Suzuki H, Yano M, Endo T, et al. (2010). Implications of serum bone turnover markers in prostate cancer patients with bone metastasis. Urology 75: 1446-1451. http://dx.doi.org/10.1016/j.urology.2009.11.049

Kataoka A, Yuasa T, Kageyama S, Tsuchiya N, et al. (2006). Diagnosis of bone metastasis in men with prostate cancer by measurement of serum ICTP in combination with alkali phosphatase and prostate-specific antigen. Clin. Oncol. (R. Coll. Radiol.) 18: 480-484. http://dx.doi.org/10.1016/j.clon.2006.02.010

Kitajima K, Murphy RC, Nathan MA, Froemming AT, et al. (2014). Detection of recurrent prostate cancer after radical prostatectomy: comparison of 11C-choline PET/CT with pelvic multiparametric MR imaging with endorectal coil. $J$. Nucl. Med. 55: 223-232. http://dx.doi.org/10.2967/jnumed.113.123018

Koo KC, Park SU, Kim KH, Rha KH, et al. (2015). Predictors of survival in prostate cancer patients with bone metastasis and extremely high prostate-specific antigen levels. Prostate Int. 3: 10-15. http://dx.doi.org/10.1016/j.prnil.2015.02.006

Lein M, Wirth M, Miller K, Eickenberg HU, et al. (2007). Serial markers of bone turnover in men with metastatic prostate cancer treated with zoledronic Acid for detection of bone metastases progression. Eur. Urol. 52: 1381-1387. http:// dx.doi.org/10.1016/j.eururo.2007.02.033

Lojanapiwat B, Anutrakulchai W, Chongruksut W and Udomphot C (2014). Correlation and diagnostic performance of the prostate-specific antigen level with the diagnosis, aggressiveness, and bone metastasis of prostate cancer in clinical practice. Prostate Int. 2: 133-139. http://dx.doi.org/10.12954/PI.14054

Luna A, Vilanova JC and Alcalá Mata L (2015). Total body MRI in early detection of bone metastasis and its indication in comparison to bone scan and other imaging techniques. Arch. Esp. Urol. 68: 371-390.

Mai ZP, Yan WG, Li HZ, Ji ZG, et al. (2015). Clinical characteristics and outcome of gleason score 10 prostate cancer on core biopsy treated by external radiotherapy and hormone therapy. Chin. Med. Sci. J. 30: 90-94. http://dx.doi. org/10.1016/S1001-9294(15)30018-3

Moslehi M, Cheki M, Salehi-Marzijarani M, Amuchastegui T, et al. (2013). Predictors of bone metastasis in pre-treatment staging of asymptomatic treatment-naïve patients with prostate cancer. Rev. Esp. Med. Nucl. Imagen Mol. 32: 286289.

Nguyen-Nielsen M, Liede A, Maegbaek ML, Borre M, et al. (2015). Survival and PSA-markers for mortality and metastasis in nonmetastatic prostate cancer treated with androgen deprivation therapy. Cancer Epidemiol. 39: 623632. http://dx.doi.org/10.1016/j.canep.2015.05.008

Pasoglou V, Michoux N, Peeters F, Larbi A, et al. (2015). Whole-body 3D T1-weighted MR imaging in patients with prostate cancer: feasibility and evaluation in screening for metastatic disease. Radiology 275: 155-166. http://dx.doi. org/10.1148/radiol.14141242

Ramankulov A, Lein M, Kristiansen G, Loening SA, et al. (2007). Plasma osteopontin in comparison with bone markers as indicator of bone metastasis and survival outcome in patients with prostate cancer. Prostate 67: 330-340. http:// dx.doi.org/10.1002/pros.20540

Szot W, Kostkiewicz M, Zając J, Owoc A, et al. (2014). Prostate cancer in patients from rural and suburban areas--PSA value, Gleason score and presence of metastases in bone scan. Ann. Agric. Environ. Med. 21: 888-892. http://dx.doi. org/10.5604/12321966.1129953

Takagi T, Katagiri H, Kim Y, Suehara Y, et al. (2015). Skeletal metastasis of unknown primary origin at the initial visit: a retrospective analysis of 286 cases. PLoS One 10: e0129428. http://dx.doi.org/10.1371/journal.pone.0129428

Wang Y, Zhang XF, Dai J, Zheng YC, et al. (2013). Predictive value of serum bone sialoprotein and prostate-specific antigen doubling time in patients with bone metastasis of prostate cancer. J. Huazhong Univ. Sci. Technol. Med. Sci. 33: 559-562. http://dx.doi.org/10.1007/s11596-013-1158-z 